\title{
BMJ
}

\section{Cluster randomised trial of a targeted multifactorial intervention to prevent falls among older people in hospital}

\author{
Robert G Cumming, professor of epidemiology and geriatric medicine, ${ }^{1,2}$ Catherine Sherrington, research \\ fellow, ${ }^{1,3,4}$ Stephen R Lord, senior principal research fellow, ${ }^{4}$ Judy M Simpson, professor (biostatistics), ${ }^{1}$ \\ Constance Vogler, staff specialist geriatrician, ${ }^{5}$ Ian D Cameron, professor of rehabilitation medicine, ${ }^{6}$ \\ Vasi Naganathan, senior lecturer geriatric medicine, ${ }^{2}$ for the Prevention of Older People's Injury Falls \\ Prevention in Hospitals Research Group
}

${ }^{1}$ School of Public Health, University of Sydney, Sydney, Australia

${ }^{2}$ Centre for Education and Research on Ageing, Concord Hospital, Australia

${ }^{3}$ Faculty of Health Sciences, University of Sydney, Lidcombe, Australia

${ }^{4}$ Prince of Wales Medical Research Institute, University of New South Wales, Kensington, Australia

${ }^{5}$ Department of Aged Care and Rehabilitation, Royal North Shore Hospital, St Leonards, Australia

${ }^{6}$ Rehabilitation Studies Unit, University of Sydney, Ryde, Australia

Correspondence to: R G Cumming, Centre for Education and Research on Ageing, Concord Hospital, Concord, NSW 2139, Australia bobc@health.usyd.edu.au

doi:10.1136/bmj.39499.546030.BE

\section{ABSTRACT}

Objective To determine the efficacy of a targeted multifactorial falls prevention programme in elderly care wards with relatively short lengths of stay.

Design Cluster randomised trial.

Setting 24 elderly care wards in 12 hospitals in Sydney, Australia.

Participants 3999 patients, mean age 79 years, with a median hospital stay of seven days.

Interventions A nurse and physiotherapist each worked for 25 hours a week for three months in all intervention wards. They provided a targeted multifactorial intervention that included a risk assessment of falls, staff and patient education, drug review, modification of bedside and ward environments, an exercise programme, and alarms for selected patients.

Main outcome measure Falls during hospital stay. Results Intervention and control wards were similar at baseline for previous rates of falls and individual patient characteristics. Overall, 381 falls occurred during the study. No difference was found in fall rates during followup between intervention and control wards: respectively, 9.26 falls per 1000 bed days and 9.20 falls per 1000 bed days $(P=0.96)$. The incidence rate ratio adjusted for individual lengths of stay and previous fall rates in the ward was 0.96 (95\% confidence interval 0.72 to 1.28 ). Conclusion A targeted multifactorial falls prevention programme was not effective among older people in hospital wards with relatively short lengths of stay. Trial registration Australian New Zealand Clinical Trials Registry ACTRNO 12605000467639.

\section{INTRODUCTION}

Falls are a serious problem among older people, with $30 \%$ of people aged 65 years or more falling at least once a year. Good evidence shows that many falls among older people living in the community can be prevented. ${ }^{1}$ Effective interventions include multifactorial falls prevention programmes and individual interventions such as exercise programmes, modifications to the home, cataract surgery, and reduced use of psychotropic drugs.
Falls also occur often among older people in hospital. Published fall rates among older in-patients vary from three to 20 per 1000 bed days, with between 3\% and $20 \%$ of patients falling at least once during their hospital stay.$^{2-6}$ Falls in hospital often result in injuries, increased lengths of stay, and greater costs to the health service. ${ }^{6-9}$

Evidence on the best way to prevent falls in hospital is, however, limited. No single intervention, such as bed alarms or bracelets to identify patients at high risk of falls, has been proved effective in randomised trials. ${ }^{10}$ In a recently published systematic review of multifaceted programmes for falls prevention in hospital, ${ }^{10}$ only two of 13 studies were properly randomised trials of such interventions. ${ }^{1112}$ Both trials found a reduction in falls but in one this was only among patients with hospital stays of 45 days or more,${ }^{11}$ and the other trial had methodological problems, with only eight wards involved and an unconventional statistical analysis. ${ }^{12}$ Methodologically sound randomised trials of interventions to prevent falls for patients in more typical elderly care wards with lengths of stay of just one or two weeks are needed.

We carried out a large cluster randomised trial of a multifactorial intervention programme for falls prevention targeted at acute and rehabilitation elderly care wards in hospitals in Sydney, Australia. As some aspects of the intervention, such as education of staff and modifications to the environment, were designed to effect change across whole wards we used a cluster design.

\section{METHODS}

Between October 2003 and October 2006 we carried out a cluster randomised controlled trial of 24 hospital wards in Sydney, Australia. Each ward was studied for three months. Pairs of wards participated in the study consecutively over 36 months.

\section{Ward and participant selection}

We first gathered information on type of ward (acute elderly care or rehabilitation), fall rates, lengths of stay, and patients' ages from most of the acute and 


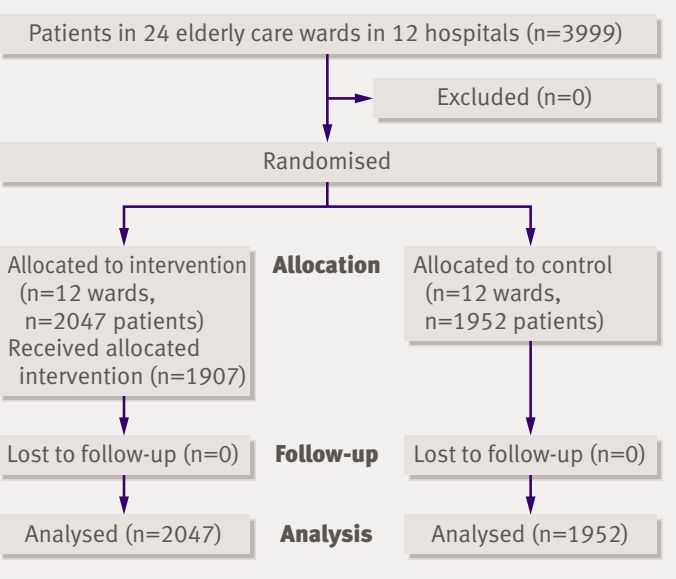

Flow of wards and participants through trial

rehabilitation elderly care wards in Sydney so that we could match pairs of similar wards before randomisation. We initially attempted to identify pairs of wards in the same hospital that were similar on the four matching characteristics. This was possible for nine pairs of wards in eight hospitals, with one hospital having two pairs of similar wards. The remaining three pairs of wards were spread across four hospitals. The study involved 24 elderly care wards in 12 hospitals: four wards in one hospital, three wards in one hospital, two wards each in seven hospitals (in one of these hospitals the two wards could not be matched with each other), and one ward each in three hospitals.

We included all patients in study wards during each three month study period. Research assistants collected baseline information on health, drugs, and physical function from the medical records of all patients in intervention and control wards, for descriptive purposes only. These research assistants had no direct

\begin{tabular}{|c|c|c|}
\hline Characteristics & Intervention wards $(\mathrm{n}=2047)$ & Control wards ( $n=1952)$ \\
\hline Mean (SD) age (years) & $79.6(12.3)$ & $78.4(13.2)$ \\
\hline Women & $1258(61)$ & $1118(57)$ \\
\hline Resident in aged care facility & $451(22)$ & $420(22)$ \\
\hline \multicolumn{3}{|l|}{ Mobility: } \\
\hline Bed rest & $268(14)$ & $305(17)$ \\
\hline Needs help to stand & $190(10)$ & $118(7)$ \\
\hline Needs help to walk & $1012(54)$ & $969(54)$ \\
\hline Walks unaided & $414(22)$ & $402(22)$ \\
\hline \multicolumn{3}{|l|}{ No of drugs: } \\
\hline $0-4$ & $354(17)$ & $363(19)$ \\
\hline $5-9$ & $1115(54)$ & $1092(56)$ \\
\hline$\geq 10$ & $578(28)$ & $496(25)$ \\
\hline \multicolumn{3}{|l|}{ Medical history: } \\
\hline Arthritis & $696(34)$ & $569(29)$ \\
\hline Dementia or confusion & $764(37)$ & $666(34)$ \\
\hline Heart disease & $1133(55)$ & $1052(54)$ \\
\hline Hip fracture & $236(12)$ & $214(11)$ \\
\hline Stroke & $379(19)$ & $366(19)$ \\
\hline
\end{tabular}

contact with patients. They could not be kept blind to whether patients were in intervention or control wards.

\section{Randomisation}

Randomisation of each matched pair of wards was usually done during the week before the study started for that pair of wards. Randomisation involved sealed, opaque envelopes and was supervised by a study investigator (usually RGC) unaware of ward characteristics.

\section{Interventions}

The intervention package was delivered by a part time nurse and a part time physiotherapist, each of whom worked 25 hours a week, mainly between 8 am and 4 pm. We chose this level of staff dedicated to falls prevention because the investigators judged that it represented the maximum feasible increase in staff in the normal (non-research) hospital setting. The same nurse and physiotherapist provided the interventions for the three years of the project. Interventions were selected from recommendations in published sources, ${ }^{13-15}$ provided they could be implemented with the resources available for this study (extra staff time and alarms). The study nurse, who had 10 years' experience in the care of elderly people, saw patients in intervention wards and carried out a falls risk assessment using a modified version of the tool developed by the Centre for Education and Research on Ageing in Sydney, Australia. ${ }^{13}$ Patients were usually assessed within 24 hours of admission, but patients admitted at weekends were not assessed until the Monday. On the basis of her assessment, the study nurse educated the patients and their families; arranged appropriate walking aids (in conjunction with the study physiotherapist), eyewear, modifications to the bedside environment, and increased supervision; and liaised with other staff about possible changes to drugs, management of confusion, and foot problems. She also ran education sessions for groups of staff and for individual members of staff.

The study physiotherapist, who had 12 years' experience in the care of elderly people, saw patients referred by the study nurse and ward staff. She supervised patients doing exercises, either individually or in groups. These exercises were designed to enhance balance and abilities with functional tasks and were in addition to any interventions provided by ward physiotherapy staff. The study physiotherapist also worked with patients to practise safe mobility within the ward environment. In conjunction with the nurse she educated staff and patients and their families about safe mobility in the ward, particularly the use of walking aids and the need for supervision.

Custom designed alarms were used for ambulant patients who staff considered to be unsafe to walk unsupported but who were likely to attempt to do so, primarily as a result of delirium or cognitive impairment. The alarm consisted of a neoprene rubber sock with a pressure switch under the heel and a small loud speaker in a pocket of the sock at ankle level. Patients 
$\overline{\text { Table } 2 \text { | Characteristics of intervention and controlwards. Values are means (standard deviations) }}$

\begin{tabular}{lcc}
$\begin{array}{l}\text { Characteristic } \\
\text { Mean (range) No of patients: }\end{array}$ & $\begin{array}{c}\text { Intervention wards } \\
(\mathrm{n}=12)\end{array}$ & Control wards $(\mathrm{n}=12)$ \\
\hline Acute elderly care wards & $228(113-330)$ & $238(121-332)$ \\
\hline Rehabilitation wards & $114(60-170)$ & $87(56-119)$ \\
\hline Lengths of stay: & & \\
\hline Acute elderly care wards & $8.5(1.7)$ & $16.8(3.7)$ \\
\hline Rehabilitation wards & $16.5(3.6)$ & \\
\hline Fall rates per 1000 bed days in previous 3 months: & & $6.7(2.6)$ \\
\hline Acute elderly care wards & $7.1(3.1)$ & $8.6(2.9)$ \\
\hline Rehabilitation wards & $9.4(5.4)$ & \\
\hline
\end{tabular}

Total number of patients was 2794 in acute elderly care wards and 1205 in rehabilitation wards.

wore one alarm device, which emitted a high pitched wavering tone ( $5 \mathrm{KHz}$ with a $14 \mathrm{~Hz}$ modulation) when weight was put on the pressure switch by patients standing after getting out of bed or rising from a chair. This alerted staff that the patient was standing and required support. The alarms were used by a small number of patients in each ward, with a maximum of two patients at any one time.

\section{Control wards}

Control wards had no trial interventions. Senior nursing staff in control wards were aware of the study because research assistants were collecting study data.

\section{Follow-up}

The primary outcome was falls in study wards during the three month study period. A fall was defined as unintentionally coming to rest on the ground or other lower level not as a result of a major internal (for example, stroke) or external event. Information on falls was collected by research assistants in three ways: from incident reports filed in patients' medical records, from notes in medical records themselves, and by asking a senior nurse each day about any falls on the ward in the past 24 hours. Research assistants were not blind to whether a ward was an intervention or control ward.

Sample size and statistical analyses

We estimated the sample size assuming $5 \%$ of patients in control wards would fall, an average cluster size

Table 3 | Falls, injurious falls, and fractures during follow-up in patients in intervention and control wards. Values are numbers (percentages)

Variable

Intervention wards $(\mathrm{n}=2047)$

Control wards $(n=1952)$

No of falls:

\begin{tabular}{lcc}
0 & $1890(92.3)$ & $1809(92.7)$ \\
\hline 1 & $127(6.2)$ & $117(6.0)$ \\
\hline 2 & $23(1.1)$ & $18(0.9)$ \\
\hline
\end{tabular}

No of injurious falls*:

\begin{tabular}{lcc}
\hline 0 & $1974(96.4)$ & $1894(97.0)$ \\
\hline 1 & $65(3.2)$ & $53(2.7)$ \\
\hline$\geq 2$ & $8(0.4)$ & $5(0.3)$ \\
\hline No of fractures & $2(0.1)$ & $3(0.1)$ \\
\hline
\end{tabular}

*Grazes, cuts, bruises, head injuries, fractures, dislocations, and sprains. (patients per ward) of 120, and an intracluster correlation coefficient of 0.005 . To have $80 \%$ power of detecting a reduction to $2.5 \%$ of patients falling at the two sided 5\% significance level required 12 intervention and 12 control wards with a total of 2880 patients.

We did analyses at both cluster (ward) and individual (adjusted for clustering) levels. Because pairwise matching of wards for fall rates in the three months before the study period was unsuccessful we did not take matching into account in the analyses. This failure of matching occurred because the data on falls we used for matching were provided by hospitals from their routinely collected data systems and was usually many months old by the time the study started in a particular pair of wards. When we checked matching at the end of the study we were able to obtain data on the three month period exactly before the study period. These are the data on previous falls that are reported in this paper and used in relevant analyses.

We used unpaired $t$ tests in analyses at cluster level to compare fall rates during the three month study period in intervention and control wards, and multiple linear regression to adjust this comparison for fall rates in the three months before the study period. For analyses of fall rates at individual level we used negative binomial regression to allow for clustering of falls by the same patient, with generalised estimating equations to allow for clustering by ward, using an exchangeable correlation structure. Models were fit first without adjustment, and then with adjustment for individual length of stay and the rate of falls in the ward in the three months before the intervention. Length of stay was calculated as only the number of days a patient was in the ward during the study period. Exploratory subgroup analyses were done using a test for interaction between subgroup and intervention group. All analyses were carried out using SAS 9.1 software.

\section{RESULTS}

The study involved 3999 patients in 24 hospital wards: 12 acute and 12 rehabilitation elderly care wards (figure). The average total number of patients per ward during the three month study period was 167 overall, 233 (range 113-332) for acute wards and 100 (range 56170) for rehabilitation wards.

In total, 381 falls occurred during the study period. Twelve (seven on intervention wards and five on control wards) were not recorded in patients' medical records and were only identified through daily verbal reports from ward staff. The overall rate of falls during the study was 9.2 per 1000 bed days, with no difference between acute wards ( 9.4 falls per 1000 bed days) and rehabilitation wards (9.0 falls per 1000 bed days). Just over $7 \%$ of patients fell at least once: $6.1 \%$ in acute wards and $10.7 \%$ in rehabilitation wards. Falls occurred most often in patients' rooms (76\% of falls), with another $11 \%$ occurring in bathrooms. Twenty eight per cent of falls occurred during the night shift (10 pm to 7 am). The most common activities at the time of a fall 
were walking $(36 \%)$ or standing from a sitting position $(24 \%)$

Patients in intervention and control wards had similar baseline characteristics (table 1). Matching of pairs of wards for previous fall rates was not successful, however, with only six pairs of wards having previous fall rates within three falls per 1000 bed days of each other. Nevertheless, overall the average fall rates in the previous three months in intervention and control wards were similar: 8.25 per 1000 bed days and 7.62 per 1000 bed days, respectively (table 2). The number of patients and mean length of stay were similar for intervention and control wards during the study (table 2).

The study nurse carried out a full assessment and developed an intervention plan for 1907 (93\%) of the 2047 patients in intervention wards. One hundred and forty patients were not seen because they were admitted and discharged outside the 25 hours worked by the study nurse. The study physiotherapist did a full assessment and tailored interventions for 884 patients (43\% of those in intervention wards). Alarms were recommended for 49 patients and 40 complied with their use.

Intervention and control wards did not differ in the frequency of falls, injurious falls, or fractures during follow-up (table 3). The mean fall rate in intervention wards was 9.26 per 1000 bed days compared with 9.20 per 1000 bed days in control wards $\left(\mathrm{t}_{22}=0.05, \mathrm{P}=0.96\right)$. After adjusting for fall rates in the wards in the three months before the study, the mean fall rates were 9.19 per 1000 bed days in intervention wards and 9.27 per 1000 bed days in control wards $(\mathrm{P}=0.95)$. Table 4 shows the results of statistical analyses at individual level using negative binomial regression. The incidence rate ratios, which give the ratio of the fall rate in intervention wards to that in control wards, were all close to 1.0, with confidence intervals excluding any major effect of the intervention in all wards combined, in acute wards only, and in rehabilitation wards only.

Table $4 \mid$ Incidence rate ratios for falls in all wards, in acute elderly care wards only, and in rehabilitation wards only

\begin{tabular}{|c|c|c|c|}
\hline Variable & $\begin{array}{l}\text { Intracluster } \\
\text { correlation } \\
\text { coefficient }\end{array}$ & $\begin{array}{l}\text { Incidence rate ratio* }(95 \% \\
\mathrm{Cl})\end{array}$ & $P$ value \\
\hline \multicolumn{4}{|l|}{ All wards: } \\
\hline Unadjusted & 0.014 & $1.02(0.70$ to 1.49$)$ & 0.92 \\
\hline $\begin{array}{l}\text { Adjusted for length of } \\
\text { stay and previous falls }\end{array}$ & 0.003 & $0.96(0.72$ to 1.28$)$ & 0.78 \\
\hline \multicolumn{4}{|l|}{ Acute elderly care wards: } \\
\hline Unadjusted & 0.007 & $1.06(0.63$ to 1.76$)$ & 0.83 \\
\hline $\begin{array}{l}\text { Adjusted for length of } \\
\text { stay and previous falls }\end{array}$ & 0.001 & 0.96 (0.68 to 1.37$)$ & 0.83 \\
\hline \multicolumn{4}{|l|}{ Rehabilitation wards: } \\
\hline Unadjusted & 0.002 & $0.92(0.64$ to 1.32$)$ & 0.64 \\
\hline $\begin{array}{l}\text { Adjusted for length of } \\
\text { stay and previous falls }\end{array}$ & 0.006 & $0.95(0.65$ to 1.40$)$ & 0.81 \\
\hline
\end{tabular}

All models are based on negative binomial regression using generalised estimating equations to adjust for clustering. *Ratio of fall rate in intervention wards to rate in control wards.
Exploratory analyses were carried out restricted to falls that occurred between 8 am and 4 pm, when study staff were most likely to be on the ward; falls that occurred in the second and third months of the intervention period, by which time usual ward staff might have become more involved in falls prevention activities; and falls occurring among patients staying in hospital more than 10 days. The intervention was not effective in any of these groups of patients (data not shown).

The intervention had no effect on rates of injurious falls (table 3). The unadjusted incidence rate ratio for injurious falls was 1.12 (95\% confidence interval 0.71 to 1.77); adjusting for length of stay and previous fall rates gave the same result. Two fractures occurred in intervention wards and three in control wards.

\section{DISCUSSION}

A targeted multifactorial intervention for prevention of falls had no effect on fall rates in elderly care wards in Sydney hospitals. The lack of effect was evident in both acute and rehabilitation wards and occurred despite the planned nursing and physiotherapy interventions being successfully implemented and the alarm for selected patients being used appropriately.

\section{Comparison with other studies}

A recent systematic review of 13 studies using multifaceted interventions in hospitals reported a pooled $18 \%$ relative reduction in falls that was just statistically significant. ${ }^{10}$ Five of the 13 studies were randomised trials and the remaining eight were before and after studies, using historical data on falls for comparison. We consider that only two of the 13 studies were properly randomised trials of truly multifactorial interventions for preventing falls in hospital. The intervention in one study was a bracelet for identifying falls, ${ }^{16}$ the falls in one study mainly occurred after discharge from hospital, ${ }^{17}$ and one study was not a randomised trial. ${ }^{18}$ This left two relevant randomised trials, both of which seemed to show a reduction in risk of falls. ${ }^{11} 12$

One of these two studies was an individually randomised trial carried out in a rehabilitation hospital in Melbourne, Australia. ${ }^{11}$ The intervention comprised a risk assessment of falls, alert cards for risk of falls, an exercise programme (which included Tai Chi), an education programme led by an occupational therapist, and hip protectors. The average length of stay was 30 days and the intervention was effective only among people with hospital stays over 45 days.

The other study was a cluster randomised trial involving eight elderly care wards in one UK hospital. ${ }^{12}$ The randomisation was unsuccessful in producing similar preintervention mean fall rates in intervention (14.4 per 1000 bed days) and control wards (18.0 per 1000 bed days). Patients who fell while in hospital were screened for falls risk factors and appropriate interventions were then implemented. These interventions included eyesight checks, drug reviews, management of postural hypotension, referral to physiotherapy, 


\section{WHAT IS ALREADY KNOWN ON THIS TOPIC}

Two randomised trials of older people in subacute and rehabilitation wards with long average lengths of stay suggest that multifactorial interventions can reduce the risk of falls

The efficacy of this approach in acute elderly care wards and short stay rehabilitation wards is uncertain

\section{WHAT THIS STUDY ADDS}

A targeted multifactorial falls prevention programme was not effective for older people in hospital wards with short lengths of stay (median seven days)

Innovative approaches to falls prevention in this setting are required

lowering bed height, moving beds nearer the nurses' station, and checks of the bedside environment. The mean length of stay was 20 days. The investigators analysed their data in an unconventional manner by calculating changes in fall rates before and during the study period and comparing the change in intervention and control wards. A $21 \%$ reduction in falls occurred in intervention wards compared with a $12 \%$ increase in falls in control wards; the difference between these changes was statistically significant $(\mathrm{P}=0.006)$. In contrast, the rate of injurious falls increased $14 \%$ in intervention wards and declined by $15 \%$ in control wards; however, this difference was not statistically significant $(\mathrm{P}=0.26)$.

Why did these two previous studies find an effect whereas ours did not? One clue is the relatively long average lengths of stay in the two previous studies ( 30 days and 20 days). In our study the median length of stay was seven days, similar to that in most modern acute care hospitals. It is likely that some falls prevention interventions (for example, exercise) need more than a few days to take effect. Another explanation for lack of effect in our study is that our intervention team might have spent too little time in each ward to effect any change in ward culture. The team spent only three months in a ward before moving on. One of the largest effect sizes in the recent systematic review was found in a study with an intervention period of two years. ${ }^{1017}$

Our intervention team spent a total of 50 person hours a week in intervention wards, mainly between 8 am and $4 \mathrm{pm}$. We chose this staffing arrangement because we wanted to evaluate an intervention that, if proved effective, could be implemented within the resource limitations of most hospitals. Although a larger input for intervention, in terms of staff time and number of staff, might have been effective in preventing falls, it is unlikely that hospitals could afford it.

\section{Strengths and limitations}

Strengths of our study include its large sample size, which resulted in fairly narrow confidence intervals ruling out the possibility that we might have missed a large intervention effect. It is possible, however, that we might have missed a small effect. The randomisation of 24 wards seems to have been successful in eliminating major systematic differences between patients in intervention and control wards. Although pairwise matching of wards by previous fall rates was not successful, the overall previous fall rates in intervention and control wards were similar. Adjusting for previous fall rates using statistical techniques had no effect on study findings.

A weakness of our study is that data were not collected blind to intervention status. It is possible that in intervention wards raised awareness of falls resulted in more diligent reporting than in control wards. Another limitation is that some falls prevention activities were already occurring in control (and intervention) wards before the start of our study. These activities would have continued during the study period, making it more difficult to show any effect of our interventions.

\section{Implications}

Our study suggests that current approaches to falls prevention in acute elderly care wards and short stay rehabilitation wards are ineffective. This may not be surprising given that the established risk factors for falls in hospital are all difficult to modify: agitated confusion, gait instability, use of sedative or hypnotic drugs, and urinary incontinence. ${ }^{19}$ Prevention of falls in hospital may require innovative approaches, including improved methods for assessing cognitive impairment, use of low beds and hip protectors to prevent injury, redesign of hospital wards so that patients at high risk of falling are readily observable at all times by busy nursing staff, 24 hour supervision of patients at highest risk of falling, and a whole system approach to ward based falls prevention led by ward staff themselves that results in changes to work practice. ${ }^{20}$

Contributors: SRL and CS had the original idea for this study. All authors contributed to the design of the study and interpretation of data. RGC wrote the first draft of the paper and all other authors provided critical intellectual input to subsequent drafts. RGC is guarantor. JMS carried out the statistical analyses of the data. All authors approved the final version. The Prevention of Older People's Injury Falls Prevention in Hospitals Research Group are: Steering Group: RGC (chair), A Broe, IDC, J Donoghue, SRL, VN, S Ogle, J Poole, R Salgado, CS, IMS, and CV. The study staff were: K Atkins, C Kirkham, S O'Rourke, and T Orr. The study sites were: Balmain Hospital (G Szonyi), Blacktown Hospital (G Cheuk), Calvary Hospital (R Salgado), Concord Hospital (VN), Greenwich Hospital (S Ni), Hornsby Kuring-gai Hospital (S Kurrle), Manly Hospital (B Chua and D Kear), Prince of Wales Hospital (A Broe and G Caplan), Royal North Shore Hospital (S Ogle, J Poole, and CV), St George Hospital (J Donoghue and P. Smerdley), War Memorial Hospital (N Brennan and S Beveridge), and Westmead Hospital (R Lindley).

Funding: This study was supported by a preventing older people's injuries grant from the National Health and Medical Research Council of Australia Health Research Partnership Scheme. All researchers are independent of the funding body.

Competing interests: None declared

Ethical approval: This study was approved by the University of New South Wales human ethics committee and by ethics committees from each involved hospital.

Provenance and peer review: Not commissioned; externally peer reviewed.

1 Gillespie LD, Gillespie WJ, Robertson MC, Lamb SE, Cumming RG, Rowe $\mathrm{BH}$. Interventions for preventing falls in elderly people. Cochrane Database Syst Rev 2006;(4):CD0003402.

2 Vassallo M, Azeem T, Pirwani MF, Sharma JC, Allen SC. An epidemiological study of falls on integrated general medical wards. Int J Clin Pract 2000;54:654-7. 
3 Vassallo M, Sharma JC, Briggs RS, Allen SC. Characteristics of early fallers on elderly patient rehabilitation wards. Age Ageing 2003;32:338-42

4 Hitcho EB, Krauss MJ, Birge S, Claiborne Dunagan W, Fischer I, Johnson S, et al. Characteristics and circumstances of falls in a hospital setting: a prospective analysis. J Gen Intern Med 2004;19:732-9.

5 Thomas E, Brennan T. Incidence and types of preventable adverse events in elderly patients: population based review of medical records. $B M J$ 2000;320:741-4.

6 Vassallo M, Sharma JC. Incidence and prognostic implications of falls associated with acute medical illness: a medical inpatient study. Int Clin Pract 1998;52:233-5

7 Nadkarni J, Iyengar K, Dussa C, Watwe S, Vishwanath K. Orthopaedic injuries following falls by hospital in-patients. Gerontology 2005;51:329-33.

8 Bates DW, Pruess K, Souney P, Platt R. Serious falls in hospitalized patients: correlates and resource utilization. Am J Med 1995;99:137-43

9 Hill KD, Vu M, Walsh W. Falls in hospital-impact on resource utilization. Aust Health Rev 2007;31:471-7.

10 Oliver D, Connelly JB, Victor CR, Shaw FE, Whitehead A, Genc Y, et al. Strategies to prevent falls and fractures in hospitals and care homes and effect of cognitive impairment: systematic review and metaanalysis. BMJ 2007;334:82-7.

11 Haines TP, Bennell KL, Osbourne RH, Hill KD. Effectiveness of targeted falls prevention programme in subacute hospital setting: randomized controlled trial. BMJ 2004;328:676-9.

12 Healey F, Monro A, Cockram A, Heseltine D. Using targeted risk factor reduction to prevent falls in older in-patients: a randomized controlled trial. Age Ageing 2004;33:390-5.
13 Shanley C. Putting your best foot forward: preventing and managing falls in aged care facilities. Sydney: Centre for Education and Research on Ageing, 1998.

14 Lord SR, Sherrington C, Menz H. Falls in older people: risk factors and strategies for prevention. Cambridge: Cambridge University Press, 2001.

15 Australian Council for Safety and Quality in Health Care. Preventing falls and harm from falls in older people. Best practice guidelines for Australian hospitals and residential aged care facilities. Canberra: Australian Council for Safety and Quality in Health Care, 2005.

16 Mayo NE, Gloutney L, Levy AR. A randomized trial of identification bracelets to prevent falls among patients in a rehabilitation hospital. Arch Phys Med Rehabil 1994;75:1302-8.

17 Reuben DB, Borok GM, Wolde-Tsadik G, Ershoff DH, Fishman LK, Ambrosini VL, et al. A randomized trial of comprehensive geriatric assessment in the care of hospitalized patients. New Engl J Med 1995;332:1345-50.

18 Vassallo M, Vignaraja R, Sharma JC, Hallam H, Binns K, Briggs R, et al. The effect of changing practice on fall prevention in a rehabilitative hospital: the Hospital Injury Prevention Study. J Am Geriatr Soc 2004;52:335-9.

19 Oliver D, Daly F, Martin F, McMurdo M. Risk factors and risk assessment tools for falls in hospital inpatients: a systematic review. Age Ageing 2004;33:122-30.

20 Fonda D, Cook J, Sandler V, Bailey M. Sustained reduction in serious fall-related injuries in older people in hospital. Med J Aust 2006;184:379-82

Accepted: 7 February 2008

o 